\title{
TWO-POINT DISTORTION THEOREMS FOR CERTAIN FAMILIES OF ANALYTIC FUNCTIONS IN THE UNIT DISC
}

\author{
YAŞAR POLATOǦLU, METIN BOLCAL, and ARZU ŞEN
}

Received 11 August 2002

\begin{abstract}
We give two-point distortion theorems for various subfamilies of analytic univalent functions. We also find the necessary and sufficient condition for these subclasses of analytic functions.
\end{abstract}

2000 Mathematics Subject Classification: 30C45.

1. Introduction. Let $\Omega$ be the family of functions $\omega(z)$ regular in the unit $\operatorname{disc} D=\{z|| z \mid<1\}$ and satisfying the conditions $\omega(0)=0,|\omega(z)|<1$ for $z \in D$.

For arbitrary fixed numbers $A, B,-1 \leq B<A \leq 1$, denote by $P(A, B)$ the family of functions

$$
p(z)=1+p_{1} z+p_{2} z^{2}+p_{3} z^{3}+\cdots
$$

regular in $D$, such that $p(z) \in P(A, B)$ if and only if

$$
p(z)=\frac{1+A \omega(z)}{1+B \omega(z)},
$$

for some functions $\omega(z) \in \Omega$ and every $z \in D$. This class was introduced by Janowski [6].

Moreover, let $C(A, B, b)$ denote the family of functions

$$
f(z)=z+a_{2} z^{2}+a_{3} z^{3}+\cdots
$$

regular in $D$, such that $f(z) \in C(A, B, b)$ if and only if

$$
1+\frac{1}{b} z \frac{f^{\prime \prime}(z)}{f^{\prime}(z)}=\frac{1+A \omega(z)}{1+B \omega(z)},
$$

where $b \neq 0, b$ is a complex number, for some functions $p(z) \in P(A, B)$ and all $z \in D$.

Next we consider the following class of functions defined in $D$.

Let $S^{*}(A, B, b)$ denote the family of functions

$$
f(z)=z+b_{1} z+b_{2} z^{2}+b_{3} z^{3}+\cdots
$$


regular in $D$, such that $f(z) \in S^{*}(A, B, b)$ if and only if

$$
1+\frac{1}{b}\left(z \frac{f^{\prime}(z)}{f(z)}-1\right)=\frac{1+A \omega(z)}{1+B \omega(z)}
$$

where $b \neq 0, b$ is a complex number, for some functions $\omega(z) \in \Omega$ and all $z \in D$.

We obtain the following subclasses of $C(A, B, b)$ by giving specific values to $A, B$, and $b$. For $A=1, B=-1$, and $b=1, C(1,-1,1)$ is the well-known class of convex functions [3, 4]. For $A=1, B=-1$, and $b=1-\alpha(0 \leq \alpha<$ $1), C(1,-1,1-\alpha)$ is the class of convex functions of order $\alpha$ introduced by Robertson [9].

For $A=1, B=-1, C(1,-1, b)$ is the class of convex functions of complex order; this class was introduced by Wiatrowski [12]. For $A=1, B=-1$, and $b=e^{-i \lambda} \cos \lambda,|\lambda|<\pi / 2, C\left(1,-1, e^{-i \lambda} \cos \lambda\right)$ is the class of functions for which $z f^{\prime}(z)$ is $\lambda$-spirallike; this class was introduced by Robertson [10].

For $A=1, B=-1$, and $b=(1-\alpha) e^{-i \lambda} \cos \lambda, 0 \leq \alpha<1,|\lambda|<\pi / 2, C(1,-1,(1-$ $\left.\alpha) e^{-i \lambda} \cos \lambda\right)$ is the class of functions for which $z f^{\prime}(z)$ is $\lambda$-spirallike of order $\alpha[1,2,7,8,11]$.

If we write $1+(1 / b) z\left(f^{\prime \prime}(z) / f^{\prime}(z)\right)=C\left(f^{\prime}(z), f^{\prime \prime}(z), b\right)$, then we obtain the following classes:

(1) the class $C(1,0, b)$ defined by $\left|C\left(f^{\prime}(z), f^{\prime \prime}(z), b\right)-1\right|<1$,

(2) the class $C(\beta, 0, b)$ defined by $\left|C\left(f^{\prime}(z), f^{\prime \prime}(z), b\right)-1\right|<\beta, 0 \leq \beta<1$,

(3) the class $C(\beta,-\beta, b)$ defined by

$$
\left|\frac{C\left(f^{\prime}(z), f^{\prime \prime}(z), b\right)-1}{C\left(f^{\prime}(z), f^{\prime \prime}(z), b\right)+1}\right|<1, \quad 0<\beta
$$

(4) the class $C(1,(1-1 / M), b)$ defined by $\left|C\left(f^{\prime}(z), f^{\prime \prime}(z), b\right)-M\right|<M$, $M>1$.

Similarly, the subclasses of $S^{*}(A, B, b)$ are obtained by giving specific values to $A, B$, and $b$. These subclasses are obtained in $[1,2,7,8,11]$.

2. Preliminary lemmas. For the purpose of this paper, we give the following lemmas.

LEMMA 2.1. The necessary and sufficient condition for $f(z) \in C(A, B, b)$ is

$$
f(z)= \begin{cases}\int_{0}^{z}(1+B \omega(\zeta))^{b(A-B) / B} d \zeta, & B \neq 0 \\ \int_{0}^{z} e^{b A \omega(\zeta)} d \zeta, & B=0\end{cases}
$$

where $\omega(z) \in \Omega$. 
Proof. Let $B \neq 0$ and let $f(z) \in C(A, B, b)$. From the definition of the class $C(A, B, b)$, we can write

$$
1+\frac{1}{b} z \frac{f^{\prime \prime}(z)}{f^{\prime}(z)}=p(z)=\frac{1+A \omega(z)}{1+B \omega(z)} .
$$

Equality (2.2) can be written in the form

$$
\frac{f^{\prime \prime}(z)}{f^{\prime}(z)}=b(A-B) \frac{\omega^{\prime}(z)}{1+B \omega(z)}
$$

by using Jack's lemma [5]. Integrating both sides of equality (2.3), we obtain

$$
f(z)=\int_{0}^{z}(1+B \omega(\zeta))^{b(A-B) / B} d \zeta .
$$

Equality (2.4) shows that $f(z) \in C(A, B, b)$.

Conversely, if we take differentiation from equality (2.3), we obtain

$$
f^{\prime}(z)=(1+B w(z))^{b(A-B) / B} .
$$

Differentiating both sides of equality (2.5), we obtain

$$
z \frac{f^{\prime \prime}(z)}{f^{\prime}(z)}=b(A-B) \frac{z \omega^{\prime}(z)}{1+B \omega(z)} .
$$

Using Jack's lemma [5] and after the simple calculations from (2.6), we obtain

$$
1+\frac{1}{b} z \frac{f^{\prime \prime}(z)}{f^{\prime}(z)}=p(z)=\frac{1+A \omega(z)}{1+B \omega(z)} .
$$

This equality shows that $f(z) \in C(A, B, b)$. Similarly, we obtain

$$
f(z)=\int_{0}^{z} e^{b A w(\zeta)} d \zeta \Longleftrightarrow f(z) \in C(A, B, b), \quad B=0 .
$$

LEMMA 2.2. Let $f(z) \in C(A, B, b) \Rightarrow z f^{\prime}(z) \in S^{*}(A, B, b)$.

Proof. Let

$$
g(z)=z f^{\prime}(z)
$$

Taking a logarithmic derivative of (2.9), and after simple calculations, we get

$$
1+\frac{1}{b}\left(z \frac{g^{\prime}(z)}{g(z)}-1\right)=1+\frac{1}{b} z \frac{f^{\prime \prime}(z)}{f^{\prime}(z)} .
$$

This shows that the lemma is true.

LEMMA 2.3. The class $C(A, B, b)$ is invariant under the rotation so that $f\left(e^{i \alpha} z\right) \in C(A, B, b),|\alpha| \leq 1$, whenever $f(z) \in C(A, B, b)$. 
Proof. Let $g(z)=f\left(e^{i \alpha} z\right)$. After the simple calculations from this equality we get

$$
1+\frac{1}{b} z \frac{g^{\prime \prime}(z)}{g^{\prime}(z)}=1+\frac{1}{b}\left(e^{i \alpha} z\right) \frac{f^{\prime \prime}\left(e^{i \alpha} z\right)}{f^{\prime}\left(e^{i \alpha} z\right)}, \quad|\zeta|=\left|e^{i \alpha} z\right|<1
$$

This shows that the lemma is true.

We note that the class $S^{*}(A, B, b)$ is invariant under the rotation so that $f\left(e^{i \alpha} z\right) \in S^{*}(A, B, b),|\alpha| \leq 1$, whenever $f(z) \in S^{*}(A, B, b)$.

LEMMA 2.4. Let $f(z)$ be regular and analytic in $D$ and normalized so that $f(0)=0$ and $f^{\prime}(0)=1$. A necessary and sufficient condition for $f(z) \in C(A, B, b)$ is that for each member $g(z), g(z)=z+a_{2} z^{2}+\cdots$, of $S^{*}(A, B, b)$, the equation

$$
g(z)=z\left(\frac{f(z)-f(\zeta)}{z-\zeta}\right)^{2}, \quad z, \zeta \in D, z \neq \zeta, \zeta=\eta z,|\eta| \leq 1,
$$

must be satisfied.

Proof. Let $f(z) \in C(A, B, b)$, then this function is analytic, regular, and continuous in the unit disc $D$ and by using Lemmas 2.2 and 2.3, equality (2.12) can be written in the form

$$
g(z)=z\left(f^{\prime}(z)\right)^{2}
$$

Taking the logarithmic derivative from equality (2.13) and after simple calculations, we get

$$
1+\frac{1}{b} z \frac{f^{\prime \prime}(z)}{f^{\prime}(z)}=1+\frac{1}{2 b}\left(z \frac{g^{\prime}(z)}{g(z)}-1\right)=\frac{1+A w(z)}{1+B w(z)} .
$$

If we consider equality (2.14), the definition of $C(A, B, b)$, and the definition of $S^{*}(A, B, b)$, we obtain that $g(z) \in S^{*}(A, B, b)$.

Conversely, let $g(z) \in S^{*}(A, B, b)$, then on simple calculations from equality (2.12), we get

$$
1+\frac{1}{b}\left(z \frac{g^{\prime}(z)}{g(z)}-1\right)=\frac{1}{b}\left[\frac{2 z f^{\prime}(z)}{f(z)-f(\zeta)}-\frac{z+\zeta}{z-\zeta}\right]+1-\frac{1}{b}
$$

If we write

$$
F(z, \zeta)=\frac{1}{b}\left[\frac{2 z f^{\prime}(z)}{f(z)-f(\zeta)}-\frac{z+\zeta}{z-\zeta}\right]+1-\frac{1}{b}
$$

equality (2.15) can be written in the form

$$
F(z, \zeta)=1+\frac{1}{b}\left(z \frac{g^{\prime}(z)}{g(z)}-1\right) .
$$


On the other hand,

$$
\operatorname{Lim}_{\zeta \rightarrow z} F(z, \zeta)=1+\frac{1}{b} z \frac{f^{\prime \prime}(z)}{f^{\prime}(z)}=1+\frac{1}{b}\left(z \frac{g^{\prime}(z)}{g(z)}-1\right)=\frac{1+A w(z)}{1+B w(z)}
$$

Equality (2.18) shows that $f(z) \in C(A, B, b)$.

COROLlary 2.5. If $f(z) \in C(A, B, b)$, then

$$
2\left[1+\frac{1}{b}\left(z \frac{f^{\prime}(z)}{f(z)}-1\right)\right]-1=p(z)=\frac{1+A w(z)}{1+B w(z)}
$$

Proof. If we take $\zeta=0$ in $F(z, \zeta)$, we obtain

$$
F(z, 0)=\frac{1}{b}\left(2 z \frac{f^{\prime}(z)}{f(z)}-1\right)+1-\frac{1}{b}=p(z)=\frac{1+A w(z)}{1+B w(z)}
$$

This shows that the corollary is true.

COROLLARY 2.6. If $f(z) \in C(A, B, b)$, then the set of values of $\left(z\left(f^{\prime}(z) / f(z)\right)\right)$ is the closed disc with centre $C(r)$ and radius $g(r)$, where

$$
\begin{gathered}
C(r)=\frac{2-\left[2 B^{2}+|b|\left(A B-B^{2}\right)\right] r^{2}}{2\left(1-B^{2} r^{2}\right)}, \\
g(r)=\frac{|b|(A-B) r}{2\left(1-B^{2} r^{2}\right)} .
\end{gathered}
$$

The proof of this corollary is obtained by using (2.19) and the inequality

$$
\left|p(z)-\frac{1-A B r^{2}}{1-B^{2} r^{2}}\right| \leq \frac{(A-B) r}{1-B^{2} r^{2}}, \quad p(z) \in P(A, B)
$$

Inequality (2.22) was proved by Janowski [6].

LEMMA 2.7. If $f(z) \in C(A, B, b)$ and $h_{\rho}(z)$ is defined by

$$
h_{\rho}(z)=\frac{f(\rho((z+a) /(1+z \bar{a})))-f(a)}{\left(1-|a|^{2}\right) f^{\prime}(a)}, \quad a, z \in D, \quad \rho \in(0,1) \text {, }
$$

then $h_{\rho}(z) \in C(A, B, b)$. 
Proof. Let $B \neq 0$. After simple calculations from (2.23), we obtain

$$
\begin{aligned}
1+\frac{1}{b} z & \frac{h_{\rho}^{\prime \prime}(z)}{h_{\rho}^{\prime}(z)} \\
= & \frac{\left(1-|a|^{2}\right) z}{(z+a)(1+z \bar{a})}\left[1+\frac{1}{b}\left(\rho\left(\frac{z+a}{1+z \bar{a}}\right)\right) \frac{f^{\prime \prime}(\rho((z+a) /(1+\bar{z} a)))}{f^{\prime}(\rho((z+a) /(1+z \bar{a})))}\right] \\
& +\left[1-\frac{1}{b} \frac{2 z \bar{a}}{1+z \bar{a}}-\frac{\left(1-|a|^{2}\right) z}{(z+a)(1+z \bar{a})}\right] .
\end{aligned}
$$

On the other hand, if we use Lemma 2.1, we can write

$$
\frac{f(\rho((z+a) /(1+z \bar{a})))-f(a)}{\left(1-|a|^{2}\right) f^{\prime}(a)}=\int_{0}^{z}(1+B \omega(\zeta))^{b(A-B) / B} d \zeta
$$

After a brief computation from equality (2.25), we get

$$
\begin{aligned}
\frac{1+A \omega(z)}{1+B \omega(z)}= & \frac{\left(1-|a|^{2}\right) z}{(z+a)(1+z \bar{a})}\left[1+\frac{1}{b}\left(\rho\left(\frac{z+a}{1+z \bar{a}}\right)\right) \frac{f^{\prime \prime}(\rho((z+a) /(1+z \bar{a})))}{f^{\prime}(\rho((z+a) /(1+z \bar{a})))}\right] \\
& +\left[1-\frac{1}{b} \frac{2 z \bar{a}}{1+z \bar{a}}-\frac{\left(1-|a|^{2}\right) z}{(z+a)(1+z \bar{a})}\right] .
\end{aligned}
$$

Let $B=0$. Similarly,

$$
\begin{gathered}
\frac{f(\rho((z+a) /(1+z \bar{a})))-f(a)}{\left(1-|a|^{2}\right) f^{\prime}(a)}=\int_{0}^{z} e^{b A \omega(\zeta)} d \zeta \Rightarrow \frac{1+A \omega(z)}{1+B \omega(z)}=1+A \omega(z) \\
=\frac{\left(1-|a|^{2}\right) z}{(z+a)(1+z \bar{a})}\left[1+\frac{1}{b}\left(\rho\left(\frac{z+a}{1+z \bar{a}}\right)\right) \frac{f^{\prime \prime}(\rho((z+a) /(1+z \bar{a})))}{f^{\prime}(\rho((z+a) /(1+z \bar{a})))}\right] \\
\quad+\left[1-\frac{1}{b} \frac{2 z \bar{a}}{1+z \bar{a}}-\frac{\left(1-|a|^{2}\right) z}{(z+a)(1+z \bar{a})}\right] .
\end{gathered}
$$

In (2.26) and (2.27), letting $z=e^{i \theta}$ and $\omega=\rho\left(\left(e^{i \theta}+a\right) /\left(1+e^{i \theta} \bar{a}\right)\right)$ gives

$$
\begin{aligned}
\frac{1+A \omega(z)}{1+B \omega(z)}= & \frac{\left(1-|a|^{2}\right)}{\left|1+a e^{-i \theta}\right|^{2}}\left[1+\frac{1}{b} \omega \frac{f^{\prime \prime}(\omega)}{f^{\prime}(\omega)}\right] \\
& +\left[1-\frac{1}{b} \frac{2 e^{i \theta} \bar{a}}{1+e^{i \theta} \bar{a}}-\frac{\left(1-|a|^{2}\right) e^{i \theta}}{\left|1+e^{-i \theta} a\right|^{2}}\right] \\
= & 1+\frac{1}{b} z \frac{h_{\rho}^{\prime \prime}(z)}{h_{\rho}^{\prime}(z)},
\end{aligned}
$$


and we conclude that $h_{\rho}(z)$ is in (2.27) for every admissible $\rho$. From the compactness of $C(A, B, b)$ and (2.28), we infer that $h(z)=\lim _{\rho \rightarrow 1} h_{\rho}(z)$ is in $C(A, B, b)$.

3. Two-point distortion for the class $C(A, B, b)$. In this section, we give two-point distortion theorems for the class $C(A, B, b)$.

THEOREM 3.1. Let $f(z) \in C(A, B, b)$. Then for $|z|=r, 0 \leq r<1$,

$$
\begin{gathered}
\frac{(1+B|z|)^{(B-A)(|b|-\operatorname{Re} b) / 2 B}}{(1-B|z|)^{(B-A)(|b|+\operatorname{Re} b) / 2 B}} \\
\leq\left|f^{\prime}(z)\right| \leq \frac{(1-B|z|)^{(B-A)(|b|-\operatorname{Re} b) / 2 B}}{(1+B|z|)^{(B-A)(|b|+\operatorname{Re} b) / 2 B}}, \quad B \neq 0, \\
e^{-A|b||z|} \leq\left|f^{\prime}(z)\right| \leq e^{A|b||z|}, \quad B=0 .
\end{gathered}
$$

Proof. If we use the definition of the class $C(A, B, b)$, then we obtain

$$
\operatorname{Re}\left(z \frac{f^{\prime \prime}(z)}{f^{\prime}(z)}\right) \geq \frac{\operatorname{Re} b\left(B^{2}-A B\right) r^{2}-|b|(A-B) r}{1-B^{2} r^{2}}, \quad B \neq 0,
$$

since

$$
\operatorname{Re} z \frac{f^{\prime \prime}(z)}{f^{\prime}(z)}=r \frac{\partial}{\partial r} \log \left|f^{\prime}(z)\right|, \quad|z|=r
$$

and using (3.2), we obtain

$$
\frac{\partial}{\partial r} \log \left|f^{\prime}(z)\right| \geq \frac{\operatorname{Re} b\left(B^{2}-A B\right) r-|b|(A-B)}{\left(1-B^{2} r^{2}\right)} .
$$

Integrating both sides of inequality (3.4) from 0 to $r$, we obtain

$$
\left|f^{\prime}(z)\right| \geq \frac{(1+B|z|)^{(B-A)(|b|-\operatorname{Re} b) / 2 B}}{(1-B|z|)^{(B-A)(|b|+\operatorname{Re} b) / 2 B}}
$$

Similarly, we obtain the bounds on the right-hand side of (3.1).

If $B=0$, then we have

$$
-|b| A r \leq \operatorname{Re} z \frac{f^{\prime \prime}(z)}{f^{\prime}(z)} \leq|b| A r
$$


and using (3.3), we obtain

$$
-|b| A \leq \frac{\partial}{\partial r} \log \left|f^{\prime}(z)\right| \leq|b| A
$$

Integrating both sides of inequality (3.7) from 0 to $r$, we obtain the desired result.

THEOREM 3.2. If $f(z) \in C(A, B, b)$, then, for $|z|=r, 0 \leq r<1$,

$$
\begin{aligned}
& \frac{|z|(1+B|z|)^{(B-A)(|b|-\operatorname{Re} b) / 4 B}}{(1-B|z|)^{(B-A)(|b|+\operatorname{Re} b) / 4 B}} \\
& \leq|f(z)| \leq \frac{|z|(1-B|z|)^{(B-A)(|b|-\operatorname{Re} b) / 4 B}}{(1+B|z|)^{(B-A)(|b|+\operatorname{Re} b) / 4 B}}, \quad B \neq 0, \\
& \quad|z| e^{-|b| A|z| / 2} \leq|f(z)| \leq|z| e^{|b| A|z| / 2}, \quad B=0 .
\end{aligned}
$$

Proof. If we use Corollaries 2.5 and 2.6 and the definition of the classes $C(A, B, b)$ and $P(A, B)$, we can write

$$
\left|\left[2\left(1+\frac{1}{b}\left(z \frac{f^{\prime}(z)}{f(z)}-1\right)-1\right)\right]-\frac{1-A B r^{2}}{1-B^{2} r^{2}}\right| \leq \frac{(A-B) r}{1-B^{2} r^{2}}
$$

After the simple calculations from inequality (3.9), we get

$$
\operatorname{Re} z \frac{f^{\prime}(z)}{f(z)} \geq \frac{2-|b|(A-B) r-\left(2 B^{2}-\left(B^{2}-A B\right) \operatorname{Re} b\right) r^{2}}{1-B^{2} r^{2}}
$$

since

$$
\operatorname{Re} z \frac{f^{\prime}(z)}{f(z)}=r \frac{\partial}{\partial r} \log |f(z)|
$$

and using (3.10), we obtain

$$
\frac{\partial}{\partial r} \log |f(z)| \geq \frac{2-|b|(A-B) r-\left(2 B^{2}-\left(B^{2}-A B\right) \operatorname{Re} b\right) r^{2}}{2 r\left(1-B^{2} r^{2}\right)}
$$

Integrating both sides of this inequality from 0 to $r$, we obtain

$$
|f(z)| \geq \frac{|z|(1+B|z|)^{(B-A)(|b|-\operatorname{Re} b) / 4 B}}{(1-B|z|)^{(B-A)(|b|+\operatorname{Re} b) / 4 B}} .
$$

Similarly, we obtain the upper bounds in (3.8). Thus we end the proof. 
We note that the bounds in Theorems 3.1 and 3.2 are sharp because the extremal function is

$$
\begin{aligned}
f_{*}(z) & = \begin{cases}e^{A b z}, & B \neq 0, \\
\frac{z(1-B z)^{(B-A)(|b|-2 \operatorname{Re} b) / 4 B}}{(1+B z)^{(B-A)(|b|+\operatorname{Re} b) / 4 B}}, & B=0,\end{cases} \\
z & =\left(\frac{r(r-\sqrt{\bar{b} / b})}{1-r \sqrt{\bar{b} / b}}\right) .
\end{aligned}
$$

COROllary 3.3. Let $f(z) \in C(A, B, b)$. Then

$$
\begin{gathered}
\alpha F_{1}(u, v) \leq|f(u)-f(v)| \leq \alpha F_{2}(u, v), \quad B \neq 0, \\
\alpha G_{1}(u, v) \leq|f(u)-f(v)| \leq \alpha G_{2}(u, v), \quad B=0,
\end{gathered}
$$

where

$$
\begin{aligned}
\alpha & =\left(1-|v|^{2}\right) \frac{|u-v|}{|1-\bar{v} u|}, \\
F_{1}(u, v) & =\frac{(1+B|z|)^{3(B-A)(|b|-\operatorname{Re} b) / 4 B}}{(1-B|z|)^{3(B-A)(|b|+\operatorname{Re} b) / 4 B}}, \\
F_{2}(u, v) & =\frac{(1-B|z|)^{3(B-A)(|b|-\operatorname{Re} b) / 4 B}}{(1+B|z|)^{3(B-A)(|b|+\operatorname{Re} b) / 4 B}}, \\
G_{1}(u, v) & =e^{-(3 / 2)|b| A(|u-v| /|1-u \bar{v}|)}, \\
G_{2}(u, v) & =e^{(3 / 2)|b| A(|u-v| /|1-u \bar{v}|)} .
\end{aligned}
$$

Proof. If we consider Lemmas 2.1 and 2.7 and Theorem 3.2, then we can write

$$
\begin{aligned}
\frac{|z|(1+B|z|)^{(B-A)(|b|-\operatorname{Re} b) / 4 B}}{(1-B|z|)^{(B-A)(|b|-\operatorname{Re} b) / 4 B}} & \leq\left|\frac{f((z+a) /(1+z \bar{a}))-f(a)}{\left(1-|a|^{2}\right) f^{\prime}(a)}\right| \\
& \leq \frac{|z|(1-B|z|)^{(B-A)(|b|-\operatorname{Re} b) / 4 B}}{(1+B|z|)^{(B-A)(|b|+\operatorname{Re} b) / 4 B}}, \quad B \neq 0, \\
|z| e^{-|b| A|z| / 2} & \leq\left|\frac{f((z+a) /(1+z \bar{a}))-f(a)}{\left(1-|a|^{2}\right) f^{\prime}(a)}\right| \\
& \leq|z| e^{-|b| A|z| / 2}, \quad B=0 .
\end{aligned}
$$


Inequalities (3.17) can be written in the form

$$
\begin{aligned}
\left(1-|a|^{2}\right)\left|f^{\prime}(a)\right| M_{1}(|z|) & \leq\left|f\left(\frac{z+a}{1+z \bar{a}}\right)-f(a)\right| \\
& \leq\left(1-|a|^{2}\right)\left|f^{\prime}(a)\right| M_{2}(|z|), \quad B \neq 0, \\
\left(1-|a|^{2}\right)\left|f^{\prime}(a)\right| N_{1}(|z|) & \leq\left|f\left(\frac{z+a}{1+z \bar{a}}\right)-f(a)\right| \\
& \leq\left(1-|a|^{2}\right)\left|f^{\prime}(a)\right| N_{2}(|z|), \quad B=0,
\end{aligned}
$$

where

$$
\begin{aligned}
& M_{1}(|z|)=\frac{|z|(1+B|z|)^{(B-A)(|b|-\operatorname{Re} b) / 4 B}}{(1-B|z|)^{(B-A)(|b|+\operatorname{Re} b) / 4 B}}, \\
& M_{2}(|z|)=\frac{|z|(1-B|z|)^{(B-A)(|b|-\operatorname{Re} b) / 4 B}}{(1+B|z|)^{(B-A)(|b|+\operatorname{Re} b) / 4 B}}, \\
& N_{1}(|z|)=|z| e^{-|b| A|z| / 2}, \\
& N_{2}(|z|)=|z| e^{-|b| A|z| / 2} .
\end{aligned}
$$

If we take $v=a, u=(z+v) /(1+z \bar{v})$, or $z=(u-v) /(1-u \cdot \bar{v})$, and if we use Theorem 3.1 in inequalities (3.18), we obtain the desired result.

We note that these inequalities are sharp because the extremal function is

$$
f_{*}(z)= \begin{cases}e^{A b z}, & B \neq 0 \\ \frac{z(1-B z)^{(B-A)(|b|-2 \operatorname{Re} b) / 4 B}}{(1+B z)^{(B-A)(|b|+\operatorname{Re} b) / 4 B}}, & B=0 .\end{cases}
$$

\section{REFERENCES}

[1] O. Altıntaş, Ö. Özkan, and H. M. Srivastava, Majorization by starlike functions of complex order, Complex Variables Theory Appl. 46 (2001), no. 3, 207-218.

[2] O. Altıntaş and H. M. Srivastava, Some majorization problems associated with $p$ valently starlike and convex functions of complex order, East Asian Math. J. 17 (2001), no. 2, 175-183.

[3] A. W. Goodman, Univalent Functions. Volume I, Mariner Publishing, Florida, 1983.

[4]_Univalent Functions. Volume II, Mariner Publishing, Florida, 1983.

[5] I. S. Jack, Functions starlike and convex of order $\alpha$, J. London Math. Soc. (2) 3 (1971), 469-474.

[6] W. Janowski, Some extremal problems for certain families of analytic functions. I, Ann. Polon. Math. 28 (1973), 297-326.

[7] R. J. Libera, Univalent $\alpha$-spiral functions, Canad. J. Math. 19 (1967), 449-456.

[8] M. A. Nasr and M. K. Aouf, Starlike function of complex order, J. Natur. Sci. Math. 25 (1985), no. 1, 1-12.

[9] M. S. Robertson, On the theory of univalent functions, Ann. of Math. 37 (1936), 374-408.

[10] Univalent functions $f(z)$ for which $z f^{\prime}(z)$ is spirallike, Michigan Math. J. 16 (1969), 97-101. 
[11] L. Spacek, Prispevek k teorii funkci prostych, Casopis Pest. Mat. Fys. 62 (1933), 12-19.

[12] P. Wiatrowski, The coefficients of a certain family of holomorphic functions, Zeszyty Nauk. Uniw. Łódz. Nauki Mat. Przyrod. Ser. II (1971), 75-85.

Yaşar Polatoğlu: Department of Mathematics and Computer Science, Faculty of Sciences and Arts, Istanbul Kültür University, Istanbul 34191, Turkey

E-mail address: y. polatoglu@iku.edu.tr

Metın Bolcal: Department of Mathematics and Computer Science, Faculty of Sciences and Arts, Istanbul Kültür University, Istanbul 34191, Turkey

E-mail address: m. bolcal@iku.edu.tr

Arzu Şen: Department of Mathematics and Computer Science, Faculty of Sciences and Arts, Istanbul Kültür University, Istanbul 34191, Turkey 


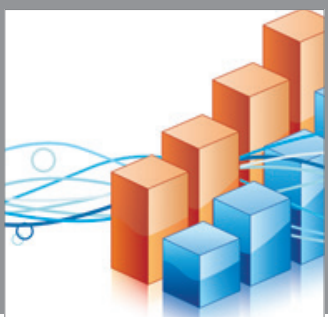

Advances in

Operations Research

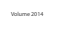

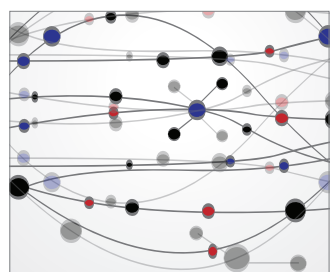

\section{The Scientific} World Journal
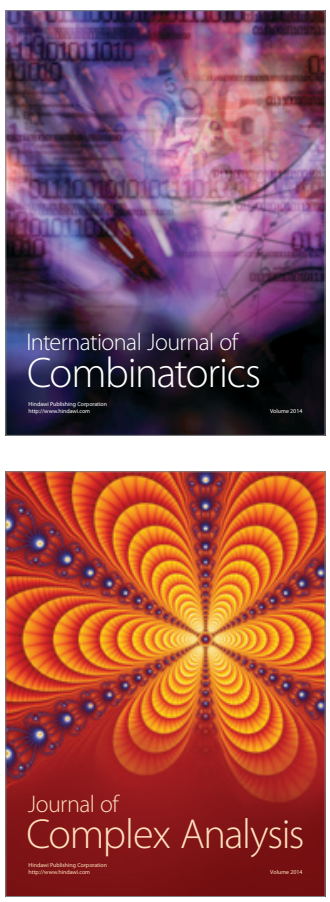

International Journal of

Mathematics and

Mathematical

Sciences
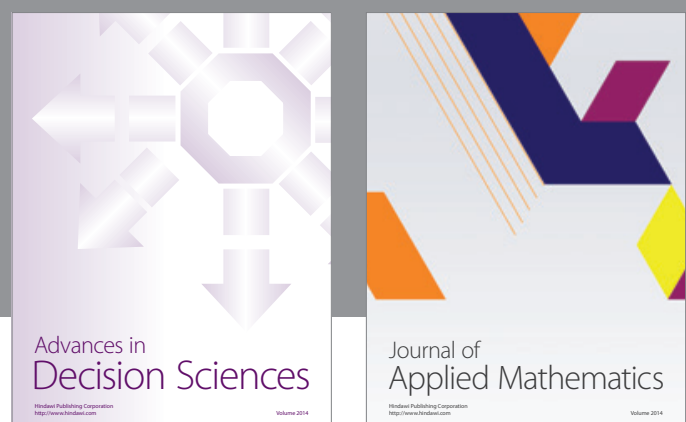

Journal of

Applied Mathematics
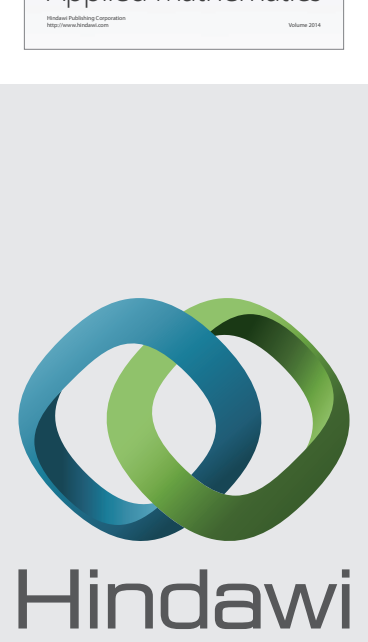

Submit your manuscripts at http://www.hindawi.com
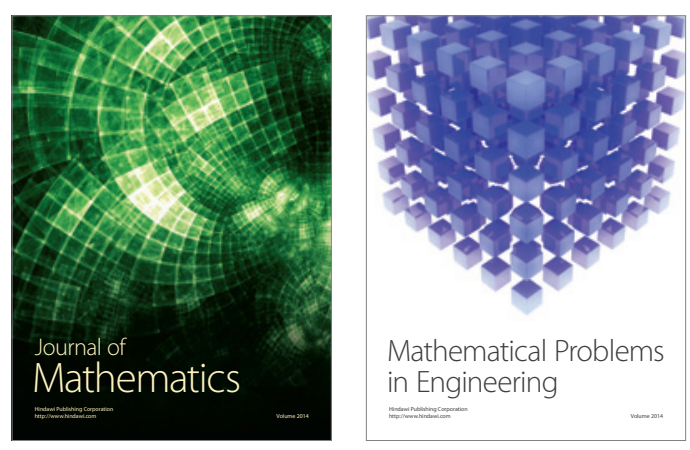

Mathematical Problems in Engineering
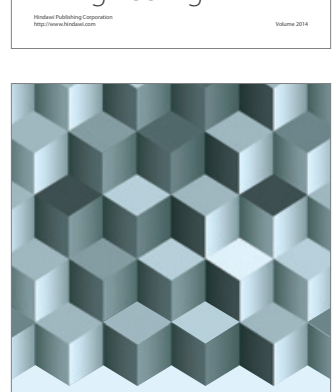

Journal of

Function Spaces
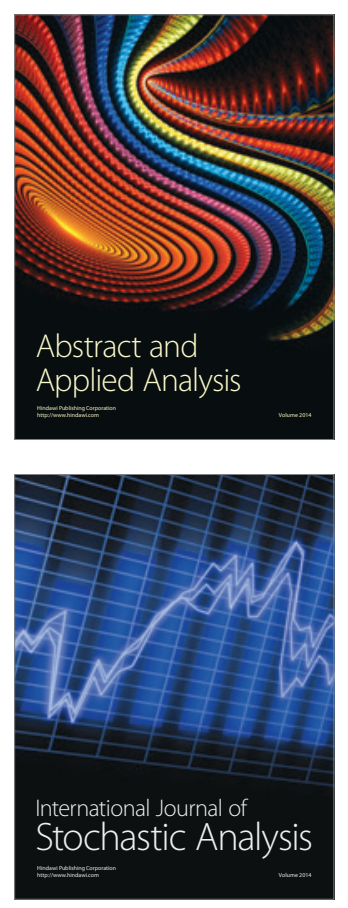

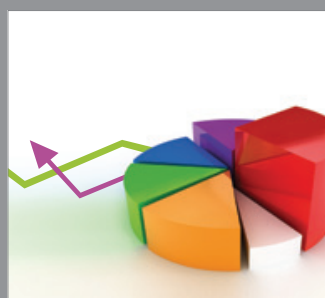

ournal of

Probability and Statistics

Promensencen
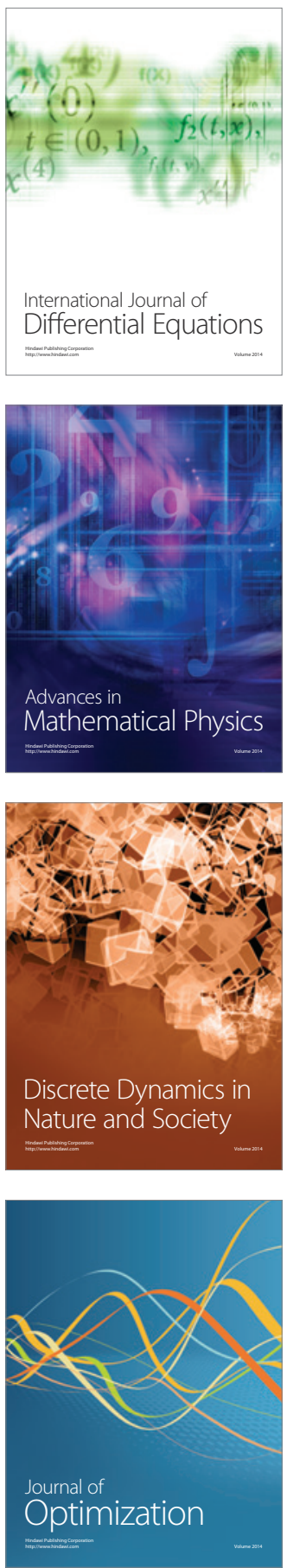